\title{
Molluscum contagiosum mimicking sebacious hyperplasia
}

\section{Bobak Hedayati', Manuel Valdebran², Amira Elbendary ${ }^{3}$, Ruzeng Xue ${ }^{4}$}

${ }^{1}$ University of California Irvine, School of Medicine, Irvine, California, USA. ${ }^{2}$ Department of Dermatology, University of California Irvine, Irvine, California. USA. ${ }^{3}$ Department of Dermatology, Kasr Alainy Faculty of Medicine, Cairo University, Egypt. ${ }^{4}$ Department of Dermatology, Dermatology Hospital, Southern Medical University, Guangzhou, China.

Corresponding author: Bobak Hedayati, E-mail: BOBAKH@hs.uci.edu

Sir,

A 61-year-old male presented to our clinic with several papules on the face for 6 months. Past medical history was unremarkable. Upon physical examination 4-7 mm yellowish dome-shape papules were found. Dermoscopy revealed umbilicated pinkish lobular structures at the center of the lesion surrounded by erythema. In addition, small radial vessels were noted. Histopathologic examination revealed dermal amphophilic squamous proliferation containing numerous eosinophilic intracytoplasmic bodies (Fig. 1).

Molluscum Contagiosum (MC) is one of the most common viral skin infections in the world. The molluscum contagiosum virus (MCV) that causes the disease, is a poxvirus that specifically targets the keratinocytes in the epidermis [1]. The virus is thought to evade the immune system, at least temporarily, as it remains superficial to the dermal-epidermal junction leading to hyperplasia of the epidermal layer. On the skin, lesions present as $3-5 \mathrm{~mm}$ skin-colored papules, each usually with a characteristic central umbilication that can arise anywhere on the body [2]. In adults, a common form of transmission is via sexual contact and as a result, lesions are often found on the genitals. However, MCV can be auto-inoculated onto other areas of the body as well [2].

Presentation in immunocompromised patients, older patients and patients with presentation in the head and neck may be difficult to diagnose clinically [3].

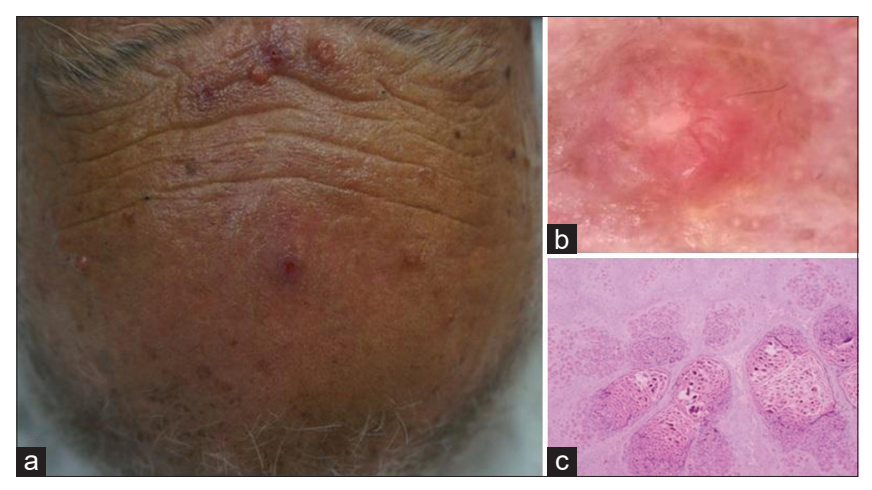

Figure 1: a) Yellowish papules with an erythematous base. b) Dermoscopy shows umbilicated lobular structures surrounded by erythema; notice small radial vessels. Dermlite DL3 x 10. c) Dermal amphophilic squamous proliferation containing numerous eosinophilic intracytoplasmic bodies. H\&E x 40 .

The dermatoscope is a useful tool for identifying the vascular pattern of MC. One study reported that roughly $89 \%$ of $\mathrm{MC}$ lesions had some type of vascular pattern which could be classified into one of four Sir,groups: crown, punctiform, radial, and mixed [4]. Sebaceous hyperplasia, has its own unique dermoscopic features including a characteristic milky, cloud-like raised globular structure with asymmetric borders and a variable vascular pattern that can be branching or nonbranching [5]. On histopathology, MC most classically presents with a central invagination that is representative of the characteristic umbilication. The keratinocytes within the lesion contain viral inclusions in the cytoplasm, called Molluscum Bodies. These can grow so large that they often displace the nuclei peripherally and can range from eosinophilic to basophilic in color. They initially are found in the keratinocytes near the basal

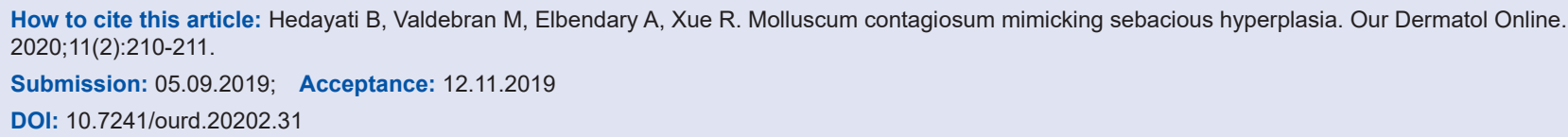


layer then progress toward the more superficial layers of the epidermis [3].

Our patient has a somewhat unique presentation for a few different reasons. First, his lesions ranged from $4-7 \mathrm{~mm}$. We observed pinkish lobular structures at the center of the lesion surrounded by erythema.

In conclusion, $\mathrm{MC}$ is a self-resolving disease in immunocompetent individuals, however, it can be spread via direct skin contact. Therefore, it is important to properly diagnose these lesions when they present. As with many diseases, MC has classic and non-classic presentations. It is imperative that physicians are aware of the less common clinical, dermatoscopic and histopathologic variations that can manifest from this disease.

\section{REFERENCES}

1. Chen X, Anstey A V, Bugert JJ. Molluscum contagiosum virus infection. Lancet Infect Dis. 2013;13:877-8.

2. Nguyen HP, Franz E, Stiegel KR, Hsu S, Tyring SK. Treatment of molluscum contagiosum in adult, pediatric, and immunodeficient populations. J Cutan Med Surg. 2014;18:299-306.

3. Ishikawa MK, Arps DP, Chow C, Hocker TL, Fullen DR. Histopathological features of molluscum contagiosum other than molluscum bodies. Histopathology. 2015;67:836-42.

4. Ianhez M, Cestari S da CP, Enokihara MY, Seize MB de PM. Padrões dermatoscópicos do molusco contagioso: estudo de 211 lesões confirmadas por exame histopatológico. An Bras Dermatol. 2011;86:74-9.

5. Bryden AM, Dawe RS, Fleming C. Dermatoscopic features of benign sebaceous proliferation. Clin Exp Dermatol. 2004;29:676-7.

Copyright by Bobak Hedayati, et al. This is an open access article distributed under the terms of the Creative Commons Attribution License, which permits unrestricted use, distribution, and reproduction in any medium, provided the original author and source are credited.

Source of Support: Nil, Conflict of Interest: None declared. 